\title{
Anharmonicity and its significance to non-Ohmic electric conduction
}

\author{
Valeri A. Makarov, ${ }^{1,2, *}$ Manuel G. Velarde, ${ }^{2, \dagger}$ Alexander Chetverikov, ${ }^{2,3, *}$ and Werner Ebeling ${ }^{2,4, \S}$ \\ ${ }^{1}$ Escuela de Optica, Universidad Complutense de Madrid, Avda. Arcos de Jalon s/n, 28037 Madrid, Spain \\ ${ }^{2}$ Instituto Pluridisciplinar, Universidad Complutense de Madrid, Paseo Juan XXIII, n. 1, 28040 Madrid, Spain \\ ${ }^{3}$ Faculty of Physics, Saratov State University, Astrakhanskaya 83, 410012 Saratov, Russia \\ ${ }^{4}$ Institut für Physik, Humboldt Universität, Newtonstrasse 15, D-12489 Berlin, Germany
}

(Received 21 December 2005; published 30 June 2006)

\begin{abstract}
We provide here a thorough analysis of the interplay between anharmonic lattice dynamics (with exponential repulsion between units) and electric conduction in a driven-dissipative electrically charged onedimensional system. First, we delineate the ranges of parameter values where, respectively, subsonic and supersonic wave solitons are possible along the lattice. Then, we study the consequences of the solitonmediated coupling of light negative to heavy positive charges (lattice units). In the presence of an external electric field we obtain the current-field characteristics for a wide range of values of all parameters defining the system. Finally, we discuss the conditions for an Ohmic-non-Ohmic transition of the electric current as the electric field strength is varied.
\end{abstract}

DOI: 10.1103/PhysRevE.73.066626

PACS number(s): 05.45.Yv, 02.30.Oz

\section{INTRODUCTION}

The experimental evidence that a polypeptide chain can form an effectively one-dimensional (1D) molecular wire appears to be highly promising for electronics at the nanoscale. For a successful approach we need a theoretical understanding of all possible electron transfer (ET) mechanisms. For instance, it has been shown that the $\mathrm{H}$ bridges contained in the protein structure play a crucial role for mediating ET. In fact the dynamics of the bonds of proteins may serve as the driving force of ET [1-7].

Some attempts to theoretically describe the charge transport in biomolecules invoked polaron and soliton models utilizing the idea that the interaction between the charge carrier and vibrational degrees of freedom of the molecular system couple together forming local compounds [8-15]. Assuming large width of the corresponding localized pulse compared to the spacing of the underlying lattice system the continuum approximation was used by Davydov and co-workers [8] to show that a mobile self-trapped state can travel as a solitary wave (soliton) along the molecular structure. In particular, charge transport in proteins was considered [9]. The soliton's stability is the result of the attained balance between two competing mechanisms, namely the interaction between an excitonic degree of freedom and the lattice vibrations of a polypeptide chain and, on the other hand, the lattice dispersion. The lattice vibrations are represented by acoustic phonon modes of not too large amplitude allowing for their description in the harmonic approximation. However, if larger displacements are to play a significant role, we ought to include higher-order terms in the amplitude of the lattice vibrations. Indeed, to obtain higher electron transport velocities necessitates so large lattice deformations that the

\footnotetext{
*Electronic address: V.A. Makarov: vmakarov@opt.ucm.es ${ }^{\dagger}$ Electronic address: M.G. Velarde: mvelarde@pluri.ucm.es

†Electronic address: A. Chetverikov: ChetverikovAP@info.sgu.ru

${ }^{\S}$ Electronic address: W. Ebeling: werner_ebeling@web.de
}

validility of the harmonic approximations does not hold anymore. Thus anharmonicity has to be included in the description of the lattice vibrations either by adding higher-order terms to the harmonic potential or considering from the beginning potentials with strong repulsive parts, represented by e.g. Lennard-Jones, Toda or Morse potentials. In the latter case, the lattice dynamics can itself support solitons which can accommodate charge with fast transfer. Noteworthy, is the much interesting recent work done on soliton-mediated electric conduction in conducting polymers [8-17].

Recently, the coupling between a quasiclassical electron dynamics and supersonic solitonic excitations in lattices with exponential repulsion has been studied. It has been shown that under special conditions the electrons might be trapped by the solitonic excitations due to the anharmonic motions of positive ions and consequent lattice deformations [18-20]. Further, a nonlinear current-voltage characteristics was derived and conditions for a transition from Ohm's linear conduction to a non-Ohmic form of conduction was predicted for a few particular values of the parameters of the system. It seems worth exploring in depth the role of anharmonic vibrations for a model problem mimicking a $1 \mathrm{D}$ electric lattice wire. The in-depth understanding of a 1D ET lattice model could give clues for the synthetic production of, e.g., a polypeptide chain built up from hydrogen-bridged peptides which may form the constituents, i.e., molecular wires of a nanoscale electronic device.

In view of the above, we provide here a thorough analysis of the dynamical system fragmentarily studied in Refs. [18-20]. A detailed discussion is given of the panoply of possibilities derived from the anharmonic character of the lattice vibrations, the variability of the stiffness of the lattice "springs," and the variable level in the input-output energy balance in the system. The present work is also a follow-up of earlier research on dynamic clustering in drivendissipative anharmonic lattices [21-24] building upon earlier results on dissipative solitons [25-27].

In Sec. II we present the model considered with the corresponding evolution equations. Section III deals with lattice vibrations and soliton waves allowed by the anharmonic dy- 


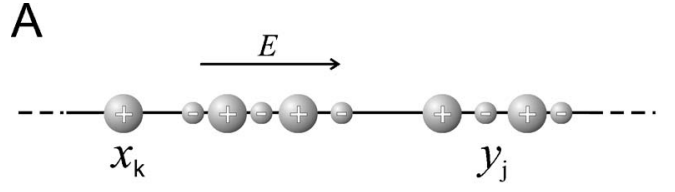

B
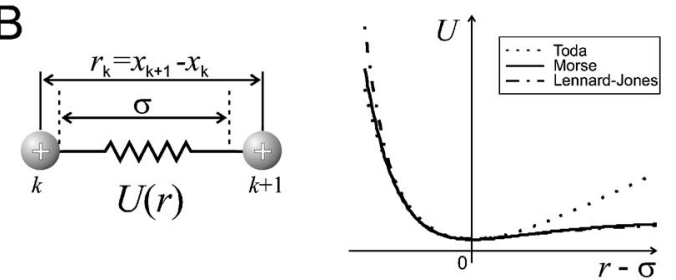

C
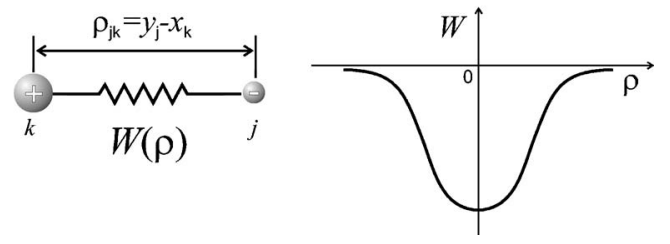

FIG. 1. Lattice dynamical system and interactions. Qualitative figures using arbitrary units: (A) Two types of charged particles of opposite sign and largely different masses, say "heavy" ions (+) and "light" electrons (-), are located on a 1D lattice, chain, with periodic boundary conditions. The particles interact among themselves and may be driven by an external, constant, homogeneous electric field $E$. An ordered motion of the particles yields an electric current. (B) Nearest-neighbor positive ions interact through an anharmonic potential $U(r)$ that depends on the interion distance $r$. The potential is strongly repulsive for ions placed below a critical distance (mean interparticle lattice distance $\sigma$ ). The Toda, Morse, and LennardJones potentials are shown for comparison. (C) The ion-electron interaction is described by an attractive Coulomb-like potential with cutoff $W(\rho)$, hence electrons are allowed to transit from one to the other side of the ions.

namics of the model system. We show that both subsonic and supersonic wave motions are possible. In Sec. IV we study the consequences of adding an external electric field, thus illustrating the possibilities of Ohmic linear conduction and non-Ohmic electric conduction for both subsonic and supersonic solitons. In the final section we highlight the results found.

\section{DYNAMICAL SYSTEM}

\section{A. Geometry, forces, and evolution equations}

Let us consider a 1D lattice of length $L$ with periodic boundary conditions [Fig. 1(A)]. This system admits two interpretations: (i) motion of particles on a ring lattice, and (ii) motion of particles on an infinite lattice with the basic cell of length $L$, which is periodically repeated. Further, we shall refer to the system simply as the ring. The ring consists of charged particles, e.g., positive ions or "dressed" ion cores. Free electrons are also added to the lattice and the action of an external, constant, homogeneous electric field $E$ is also considered. Along the ring we denote the positions of the ions by $x_{k}$, and the positions of the electrons by $y_{j}$. The ions (subscript $i$ ) are assumed to be much heavier than the electrons (subscript $e$ ), $\frac{m_{e}}{m_{i}} \simeq 10^{-3}$. To satisfy electroneutrality the number of ions and electrons on the ring is equal, $N_{i}=N_{e}$ $\equiv N$.

In equilibrium the ions initially are placed in a periodic distribution, they can oscillate around equidistant positions (spacing given by $\sigma$ ), but neither overlaps nor "crossings" are possible as we assume strongly repulsive forces for short distances. Recall that the repulsion between two spherically symmetric atoms arises when they are sufficiently close together that their electron shells (electron clouds) overlap. This is because the clouds no longer completely shield electrostatically the two nuclei from each other. A detailed quantum-mechanical calculation of such interaction is complicated and its consideration is beyond the scope of the present work. It suffices to say that, qualitatively, it is known that the charge density associated with the electrons falls off exponentially at distances comparable with the atomic radius.

The force between a pair of neighboring ions is taking from a potential $U$ that depends on the interion distance $r$ [Fig. 1(B)]. Then the complete potential of the system describing the interionic interactions is

$$
U^{\text {total }}=\sum_{k=1}^{N} U\left(r_{k}\right), \quad r_{k}=x_{k+1}-x_{k}
$$

Particular cases are the Toda and the Morse potentials with exponentially increasing repulsive forces at small distances or the Lennard-Jones (LJ) and modified Buckingham potentials. The latter combines hard-sphere and exponential repulsion with attractive power law [28-32]. In their repulsive part $(r-\sigma<0)$ these potentials exhibit similar fast growth [Fig. 1(B)]. A difference appears only in the limit of vanishing interion distance where the LJ and modified Buckingham potentials go to infinity preventing ions from overlapping, while the Toda and Morse potentials grow exponentially, remain bounded, and hence special attention should be given in numerical simulations to avoid particle overlapping or crossing when using such potentials. In the attractive part $(r-\sigma>0)$ the Toda potential is unphysical and, indeed, differs from both the Morse and LJ potentials. It is the attractive part of the Morse and LJ potentials that allows the formation of clusters of particles (condensationlike transition albeit with no true crystal ordering) in rings with low particle densities [23]. We shall not consider this case here, but rather focus attention to the region of higher densities (in equilibrium the ions are either located in the potential minimum or to the left of the minimum), where both the Morse and the LJ potentials can be well approximated by the repulsive part of the Toda potential [24]. The (infinite) lattice with Toda anharmonic "springs" is known to be able to allow different kinds of nonlinear waves including solitons [33]. We shall make use here of this possibility even though we shall be considering dissipation playing a significant role in the evolution of the dynamical system.

The Toda potential is 


$$
U^{\text {Toda }}=\frac{a}{b}\left[e^{-b(r-\sigma)}+b(r-\sigma)-1\right]
$$

where $b$ defines the stiffness of the "springs" between ions in the lattice. We shall assume that the mean interionic distance $L / N$ corresponds to the minimum position or less for a compressed lattice. Under this condition the Toda potential gives always a good approximation of the realistic potential [24]. For this reason we shall concentrate here on Toda interactions.

For the electron-ion interaction [Fig. 1(C)] we shall take an attractive Coulomb-like potential with cutoff [19,34]:

$$
W^{i-e}=q^{2} \sum_{j=1}^{N} \sum_{k=1}^{N} W\left(\rho_{j k}\right), \quad \rho_{j k}=y_{j}-x_{k},
$$

where $q$ denotes charge $(q=+e$ for ions and $q=-e$ for electrons). To eliminate unnecessary complications, the electrons are "free" to move in 3D space surrounding the 1D lattice thus mathematically simplifying the picture. We shall also require that this potential is bounded below at $\rho=0$, where it has a minimum. Avoiding the singularity at vanishing distance allows electrons to transit from one to the other side of an ion (generally free electrons are not able to penetrate "dressed" ion cores). There exist several forms of such a (pseudo)potential, for example:

$$
W= \begin{cases}- \text { const, } & \text { if }|\rho|<h \\ -\frac{1}{|\rho|}, & \text { if }|\rho|>h,\end{cases}
$$

where $h$ is a scale parameter in the dynamics. Pseudopotentials of this type are commonly used in plasma physics and in solid state theory [34-36]. Here we shall take [Fig. 1(C)]

$$
W^{C}=-\frac{1}{\sqrt{\rho^{2}+h^{2}}} .
$$

Note, however, that in the results to be discussed below the specific form of the pseudopotential plays no significant role, at least to a qualitative level.

For simplicity, we shall neglect the interaction between electrons. Such an approximation is valid in dense systems, where the electrons are subject to quantum effects (Heisenberg's uncertainty and Pauli's exclusion principle) that weaken the interelectron forces and justify the neglect of repulsion in a first approximation.

Finally, the Hamiltonian of the composite electron-lattice dynamical system is

$$
\begin{aligned}
H= & \sum_{k=1}^{N} \frac{m_{i}}{2} v_{k}^{2}+\sum_{j=1}^{N}\left(\frac{m_{e}}{2} u_{j}^{2}+U\left(r_{k}\right)\right) \\
& +\sum_{j, k=1}^{N}\left[q^{2} W\left(\rho_{j k}\right)+q E\left(y_{j}-x_{k}\right)\right],
\end{aligned}
$$

where $v$ and $u$ denote the ion and electron velocities, respectively. We have also included the contribution from the external electric field.
Following, for simplicity, the classical approach taken by Drude [35] we assume that the motion of the electrons experiences friction with the constant $\gamma_{e}$, while for the ions we shall consider a velocity-dependent friction law,

$$
f(v)=\gamma_{i} v\left(\mu_{i}-v^{2}\right) .
$$

This function (7) was first introduced by Lord Rayleigh to maintain harmonic motions in the presence of dissipation $[37,38]$. An alternative could be amplitude-square dependent friction law later on introduced by Van der Pol [39]. The sign of the bracketed quantity in Eq. (7) permits that $f(v)$ be mere friction or active friction according to the value taken by the parameter $\mu_{i}$. We shall make use here of the latter possibility when considering the system immersed in a stochastic thermal bath. The coefficient $\gamma_{i}$ is also supposed to be controllable and taken positive like $\gamma_{e}$. By means of $f(v)$ added to the dynamics (6) the nonlinear system pumps energy from the noise thus self-organizing and maintaining itself in an oscillatory mode and corresponding soliton wave along the lattice. Equation (7) or the Van der Pol alternative are not the only possible energy pumping mechanisms. Other activefriction-like dynamics are described in Ref. [40]. At the mesoscopic level, Eq. (7) with the Gaussian white noise bears similarity with a Nose-Hoover thermostat, save the specificity of acting upon individual units [41-43].

Then the equations of motion are

$$
\begin{gathered}
\frac{d x_{k}}{d t}=v_{k}, \quad \frac{d v_{k}}{d t}=-\frac{1}{m_{i}} \frac{\partial H}{\partial x_{k}}+f\left(v_{k}\right), \\
\frac{d y_{j}}{d t}=u_{j}, \quad \frac{d u_{j}}{d t}=-\frac{1}{m_{e}} \frac{\partial H}{\partial y_{j}}-\gamma u_{j} .
\end{gathered}
$$

The (mean) ordered motion of charged particles along the lattice governed by Eqs. (8) and (9) yields a current composed of ion and electron components:

$$
J=J_{i}+J_{e}=q(\langle v\rangle-\langle u\rangle) .
$$

A nonvanishing current requires different mean velocities of ions $\langle v\rangle$ and electrons $\langle u\rangle$, which is allowed due to the cutoff in the ion-electron potential [Fig. 1(C)]; $\langle w\rangle \equiv \lim \frac{1}{T} \int_{0}^{T} w d t, w$ is $u$ or $v$, for ions and electrons, respectively.

We expect two forms of electric transport in the ring: (i) For $\mu_{i}<0$, a Drude-like current, linearly increasing with the increase of the electrical field strength (Ohm's law); and (ii) for $\mu_{i}>0$, a non-Ohmic, hence nonlinear current dominated by the anharmonicity of the dynamical system, i.e., by the repulsive (Toda, Morse or LJ) short range interaction together with the active friction in dense enough lattices.

\section{B. Scales, dimensionless quantities, and approximations}

Let us introduce new units leading to dimensionless variables:

$$
t=\frac{1}{q}\left(\frac{\sigma m_{e}}{\alpha}\right)^{1 / 2} \hat{t}, \quad\left\{x_{k}, y_{j}\right\}=\sigma\left\{\hat{x}_{k}, \hat{y}_{j}\right\},
$$




$$
\left\{v_{k}, u_{j}\right\}=q\left(\frac{\alpha \sigma}{m_{e}}\right)^{1 / 2}\left\{\hat{v}_{k}, \hat{u}_{j}\right\},
$$

where $\alpha=\max \frac{\partial W}{\partial \rho}$. For illustration we shall use the Toda (2) and Coulomb (5) potentials. Dropping hats to simplify notation in dimensionless form Eqs. (8) and (9) become

$$
\begin{gathered}
\frac{d x_{k}}{d t}=v_{k}, \\
\frac{d v_{k}}{d t}=S\left(r_{k-1}\right)-S\left(r_{k}\right)+G\left(v_{k}\right)+\varepsilon\left[\sum_{j=1}^{N} F\left(\rho_{j k}\right)+\mathrm{E}\right], \\
\frac{d y_{j}}{d t}=u_{j}, \\
\frac{d u_{j}}{d t}=-\sum_{k=1}^{N} F\left(\rho_{j k}\right)-\mathrm{E}-\Gamma u_{j},
\end{gathered}
$$

with

$$
\begin{gathered}
\varepsilon=\frac{m_{e}}{m_{i}} \simeq 10^{-3}, \quad \gamma=\left(\frac{\alpha \sigma^{3}}{m_{e}}\right)^{1 / 2} q \gamma_{i}, \quad \mu=\frac{\alpha \sigma}{m_{e}} q^{2} \mu_{i}, \\
\alpha=\frac{2 \sqrt{3}}{9 h^{2}}, \quad S=\frac{1}{B} \exp [-B(r-1)], \\
F(\rho)=\frac{\rho}{\alpha\left[\rho^{2}+h^{2}\right]^{3 / 2}}, \quad \mathrm{E}=\frac{E}{\alpha q}, \quad \Gamma=\frac{\gamma_{e}}{q}\left(\frac{\sigma m_{e}}{\alpha}\right)^{1 / 2}, \\
G\left(v_{k}\right)=\gamma v_{k}\left(\mu-v_{k}^{2}\right) .
\end{gathered}
$$

Needless to say, $F(\rho)$ accounts for the attractive ion-electron force, $S(r)$ is the repulsive ion-ion force, $E$ is the dimensionless electric field strength, $\Gamma$ is the damping constant for the electrons, and $G(v)$ is the velocity-dependent friction for the ions.

Formally, $\varepsilon$ in Eqs. (12) can be treated as a smallness parameter, provided we consider that both the external field and the electron-ion interaction play a secondary role relative to the ion-ion interaction. We are aware of the subtlety of the transition $\varepsilon \rightarrow 0$ in the static case. However, when dealing with dynamical regimes, we may consider the (nonlinear) dynamics of the (heavy) ions as a suitable starting point for a perturbation theory to treat dense enough lattices when the repulsion forces are quite strong.

In the new scales the mean interion distance $(L=N)$ is unity and the ion dynamics plays a dominant role on the evolution of the lattice and the electrons, $F(\rho) \sim 1$. This biases the system to exhibit a non-Ohmic, nonlinear electric current rather than the linear Ohm-Drude current, $J_{e} \approx \frac{q}{\Gamma} \mathrm{E}$. To establish the laws of such non-Ohmic behavior, as first predicted in Ref. [18], is indeed the main object of the present work.

\section{LATTICE VIBRATIONS AND SOLITONS}

Let us, to a first approximation, neglect in Eqs. (12) the influence of the ion-electron and field-ion interactions on the ion dynamics $(\varepsilon \rightarrow 0)$. In fact let us first analyze the intrinsic lattice dynamics alone. The lattice dynamics obeys the following simplified equations:

$$
\frac{d x_{k}}{d t}=v_{k}, \quad \frac{d v_{k}}{d t}=S\left(r_{k-1}\right)-S\left(r_{k}\right)+G\left(v_{k}\right) .
$$

In an earlier work [22] it has been shown that the system in the truly damped case $(\mu<0)$ has only one motionless globally stable solution $\left\{x_{k+1}-x_{k}=1, v_{k}=0\right\}$, where all ions form an equidistant lattice.

Let us now study the role of the active friction in the ion lattice $(\mu>0)$. At $\mu=0$ the system undergoes a symmetric Hopf bifurcation [22]. The $2 N$ eigenvalues of the linearized problem are given by

$$
\delta_{m}^{1,2}=\frac{1}{2}\left(\gamma \mu \pm \sqrt{\gamma^{2} \mu^{2}-16 \sin ^{2}[\pi m / N]}\right),
$$

where $m=0, \pm 1, \ldots, \pm N / 2$ (for illustration here we assume $N$ to be even). One eigenvalue, for $m=0$, vanishes due to the translation symmetry of the system. Another is real, $\delta_{0}^{2}=\gamma \mu$, and changes sign at $\mu=0$. The other $2(N-1)$ eigenvalues are complex conjugate and cross, simultaneously, the imaginary axis at $\mu=0$, obeying the Hopf theorem [44].

For positive $\mu,(N-1)$ different oscillatory modes appear as shown in Fig. 2(A). These modes correspond to stable limit cycles coexisting in the $2 N$-dimensional phase space $\left\{r_{k}, v_{k}\right\}$ of the system. They represent ion motions leading to nonlinear waves (like acoustic modes) propagating along the lattice and can be labeled by their wave number $m$ or by their wave length $\lambda=|N / m|$; hence the single-peak soliton, $m=1$, has $\lambda=N$. The sign in the mode number defines the direction (clockwise or counterclockwise) of the wave propagation. Two other modes, say $m= \pm 0$, correspond to the clockwise and counterclockwise rotations of the ring as a whole (ions at rest). Note that since each mode corresponds to a stable limit cycle, only one mode can be realized in the ring at a time with no superposition admitted. The mode number $m$ defines the number of local compressions (wave humps, peaks, or pulses) along the ring, e.g., $m=1$ corresponds to a singlepeak soliton wave; otherwise we have a multipeak or multihump soliton wave (as described below). The $m=N / 2$ mode is the optical mode (ions moving in antiphase) [Figs. 2(B) $-2(\mathrm{D})]$.

To estimate electron transport which demands considering dissipative effects that may alter the dynamics of the ion lattice, we must know both wave and ion velocities. A hint for a theoretical estimate can be obtained numerically following the evolution when varying $B$ and $\mu$ in a wide range. In each run, Eqs. (14) were numerically integrated until a stationary wave was formed and remained practically of permanent form albeit moving along the ring. Then an estimate of the oscillation period is the time interval between two successive passes of the wave through a given ion. From bifurcation theory [44] one expects that the oscillation amplitude grows supercritically as the square root of $\mu$. Thus 
A

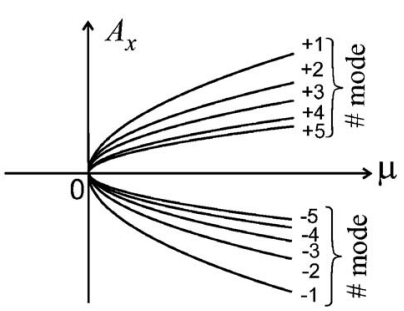

C

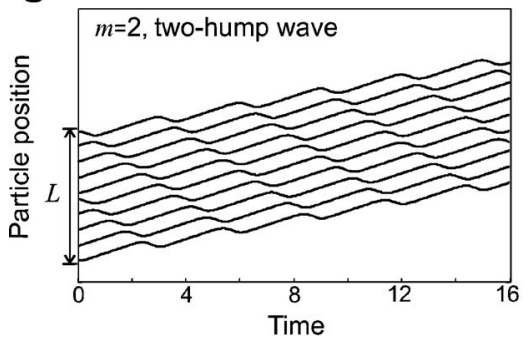

B

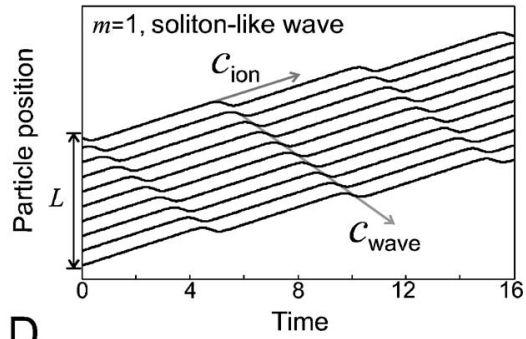

D

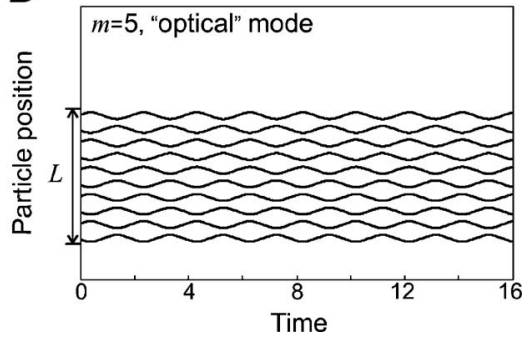

FIG. 2. Lattice vibrations and soliton waves. (A) Qualitative bifurcation diagram (amplitude of oscillations $A_{x}$ vs bifurcation parameter $\mu$, Rayleigh's active friction). The sign of $A_{x}$ is used to merely distinguish clockwise and negative from counterclockwise and positive directions of wave motion. For negative $\mu$ (truly damped case) only the motionless solution exists (with translation symmetry). At $\mu=0$ a symmetric Hopf bifurcation occurs and for $\mu>0$, the anharmonic ion vibrations lead to $(N-1)$ soliton wave modes; here $N=10$ ions. There are also two (clockwise and counterclockwise) rotatory regimes of the entire lattice as a whole. Note that the two values $m= \pm N / 2$ define the same optical mode. (B)-(D) Unfolded ion trajectories for three different wave modes moving clockwise, "downstream." In the case of a single-peak solitonlike wave $(m=1)$ most of the time ions move clockwise (hence positive slope of the trajectories) with velocity $c_{i o n}$. From time to time, sequentially one after another, ions transitorily move backwards, counterclockwise, showing the onset of the wave peak (zone of local compression). The wave moves counterclockwise, "upstream," with velocity $c_{\text {wave }}$. For $m=2$ two local compressions in a lattice period (a two-hump wave) propagate over the ring. In the optical mode $(m=N / 2=5)$ we have a standing wave, when neighboring ions oscillate in antiphase. Parameter values: $B=6, \mu=0.4, \gamma=0.4$, and $N=L=10$. Dimensionless quantities are according to Eq. (11).

for convenience we rescale once more the variables:

$$
t=T \eta, \quad v_{k}=\mu^{1 / 2} p_{k}, \quad x_{k}-x_{k-1}-1=\mu^{1 / 2} T z_{k},
$$

where $T$ denotes the period yet to be determined. The periodic boundary conditions, $x_{k+N}-x_{k}=L=N$, require that $\sum_{k=1}^{N} z_{k}=0$. Accordingly, with the new coordinates the set of variables $\{z(\xi), p(\xi)\}, \xi=k / \lambda+\eta$, depict the passage along the ring of a wave of period unity. Figure 3 shows a portion of the normalized particle velocity $p(\xi)$ and the interparticle distance $z(\xi)$ obtained by superposition of results corre-
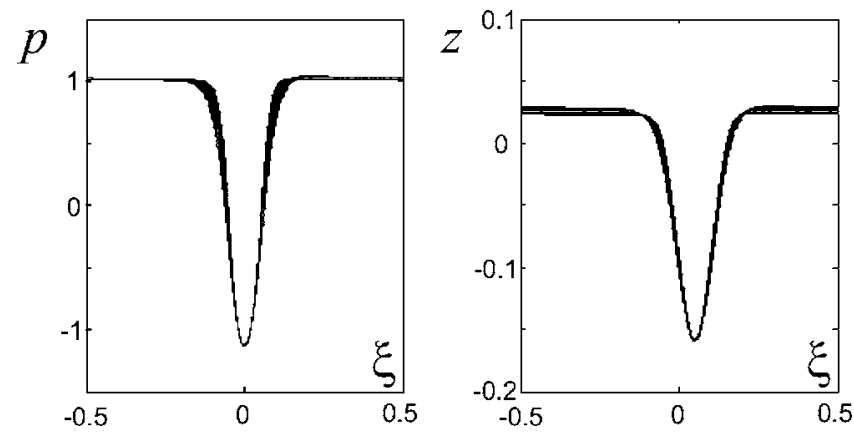

FIG. 3. Soliton wave of permanent form (mode $m=1$ ) for a wide range of parameter values $\{4 \leq B \leq 16,0.2 \leq \mu \leq 0.5\}$. Left and right figures, corresponding, respectively, to ion velocity and interionic distance come, for given values of the running coordinate $\xi$, from the superposition of a large number of computer simulations. Dimensionless quantities are according to Eq. (16). sponding to several different values of $B$ and $\mu$. In the coordinates (16) the soliton wave (single-peak or periodic wave) of permanent form, numerically found as a solution of our dissipative Toda ring, deviates little from a cnoidal wave of the conservative Toda system. Noteworthy is that it practically does not depend on the stiffness parameter $B$, nor on the pumping or bifurcation parameter $\mu$ (needless to say $\mu$ cannot be too large). The characteristic ion velocity [Fig. $2(\mathrm{~B})]$ is proportional to $\sqrt{\mu}$. Let us now estimate the wave velocity.

The period of ion oscillation is (see the Appendix):

$$
T(\mu, B, \lambda)=\frac{\pi}{\sin (\pi / \lambda)(1+\alpha \sqrt{\mu} B)},
$$

where $\lambda=N / m$ is the wavelength and $\alpha \approx 0.24$ is a constant estimated numerically.

During the oscillation period the wave travels the distance $L / m-\langle c\rangle T$, where $\langle c\rangle=\sqrt{\mu} \int_{-1 / 2}^{1 / 2} p d \xi$ is the mean ion velocity here taken over a wave period. Finally, for a counterclockwise rotating wave, $m>0$ with $c_{\text {ion }}>0$ and $c_{\text {wave }}<0$, we obtain

$$
c_{\text {ion }} \simeq \sqrt{\mu}, \quad c_{\text {wave }} \simeq-\frac{\lambda}{\pi} \sin \left(\frac{\pi}{\lambda}\right)[1+\sqrt{\mu}(\alpha B-\beta)],
$$

where $\beta(\lambda)$ is a constant. Thus for a given value of $\mu$, the wave velocity linearly increases with the increase in the stiff- 

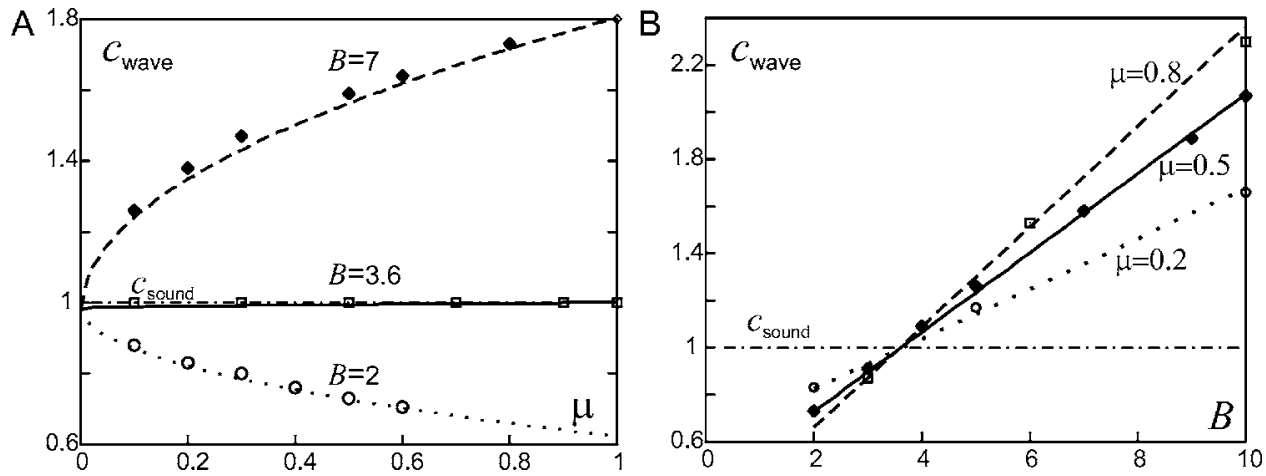

FIG. 4. Wave velocity $c_{\text {wave }}$ of the single-peak soliton wave $(m=1)$, propagating along the lattice vs bifurcation parameter $\mu$ (A) and vs lattice stiffness $B(B)$. Solid rhombs, open squares, and open circles show numerical results. Solid, dashed, and dotted lines correspond to the theoretical estimates given by Eq. (18). Noteworthy is that there are three qualitatively different functional dependences of the velocity on $\mu$ : (i) for a stiffness higher than the critical value, $B_{c r} \approx 3.6$, the velocity increases as $\sqrt{\mu}$ and it is supersonic $\left(c_{\text {sound }}=1\right.$ ), (ii) for the critical $B$ the velocity is constant $c_{\text {wave }} \approx c_{\text {sound }}$, and (iii) for small stiffness, the velocity decreases as $-\sqrt{\mu}$ and hence it is subsonic. The variation of the wave velocity with $B$ is linear with slope determined by the value of $\mu$. For the critical value of the stiffness $B_{c r} \approx 3.6$ all straight lines intersect at the sound velocity value. Parameter values: $\gamma=2$ and $L=N=10$. Dimensionless quantities are according to Eqs. (11) and (13).

ness parameter $B$. For hard "springs" the wave velocity is higher than the sound velocity; here $c_{\text {sound }}=1$. Thus we have a dissipative wave traveling supersonically along the lattice. On the other hand, for a given value of $B$, the wave velocity can either increase or decrease as the square root of $\mu$, whose value sets the level of input-output energy balance in the system. For the critical value $B_{c r}=\beta / \alpha$ the wave always runs with the velocity $c_{\text {wave }} \simeq-\frac{\lambda}{\pi} \sin \left(\frac{\pi}{\lambda}\right)$, which in the case of the single-peak soliton mode $(m=1)$, is $c_{\text {soliton }} \simeq 1$, which is about the sound velocity in the lattice. Figure 4 shows an example of numerically calculated wave velocities and the theoretical estimates given by Eq. (18), with the constant $\beta$ $=0.85$. This is at variance with the conservative case where all (single-peak or periodic) soliton waves travel supersonically along the lattice [33].

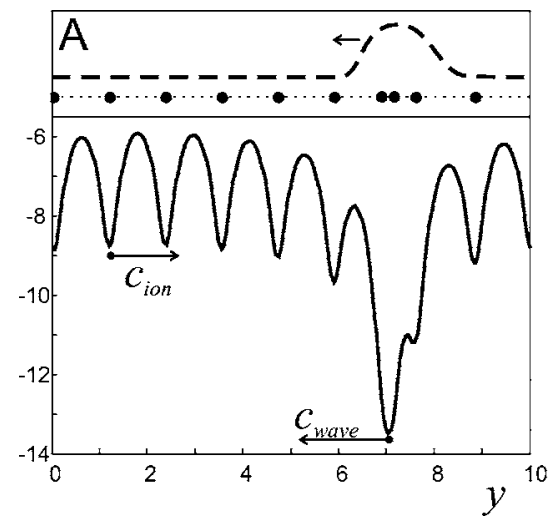

Note that not all values of the parameters $B$ and $\mu$ are physically acceptable as, to overcome the earlier mentioned difficulty with the exponential interaction here used, we must require that ions do not penetrate each other, i.e., always $x_{k+1}(t)>x_{k}(t)$. This is equivalent to $-\sqrt{\mu} T \min [z(\xi)]<1$. Using the theoretical estimate (17) we obtain that a solution of Eq. (14) has proper physical meaning only if

$$
B>\frac{\pi|\min (z)|}{\alpha \sin (\pi / \lambda)}-\frac{1}{\alpha \sqrt{\mu}} \approx 6.8-\frac{4.2}{\sqrt{\mu}} .
$$

\section{ELECTRON TRANSPORT INDUCED BY WAVES IN THE ION LATTICE}

In view of the earlier given assumptions and results, when an electric field is added, we expect that the soliton wave of

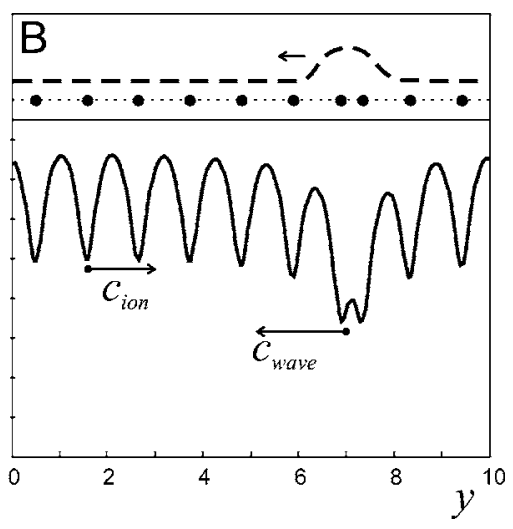

FIG. 5. Anharmonic lattice vibrations (solid dots denote ion positions along the lattice-dotted line), single-peak soliton compression wave (dashed line), and corresponding potential landscape (solid line) offered by the vibrating lattice ions to a "free" electron. (A) For a relatively weak stiffness corresponding to soft yet nonlinear "springs" $(B=1.1)$, several ions (three) transitorily cluster, rather near to each other, thus creating a deeper minimum (at $y \approx 7$ ) in the electrostatic potential offered to a free electron. Far away from the local compression ions offer a periodic landscape given by Eq. (5). As the local compression, and hence the deepest minimum, the soliton wave [see Fig. 2(B)], moves to the left, such minimum is defined by another local cluster of ions. This is clearly seen when one is placed in the ion-moving frame and then one substracts the ion velocity $c_{i o n}$ from the wave velocity $c_{\text {wave }}$. (B) For harder lattice springs $(B=7)$ ions move little from their original equilibrium positions yet the nonlinearity creates a soliton wave and a corresponding depression (at $y \approx 7$ ) in the potential landscape. However, locally, the depression zone offers two minima and a maximum to the free electron. $y$ denotes ion position along the lattice. Dimensionless velocities, according to Eqs. (13) and (18). 

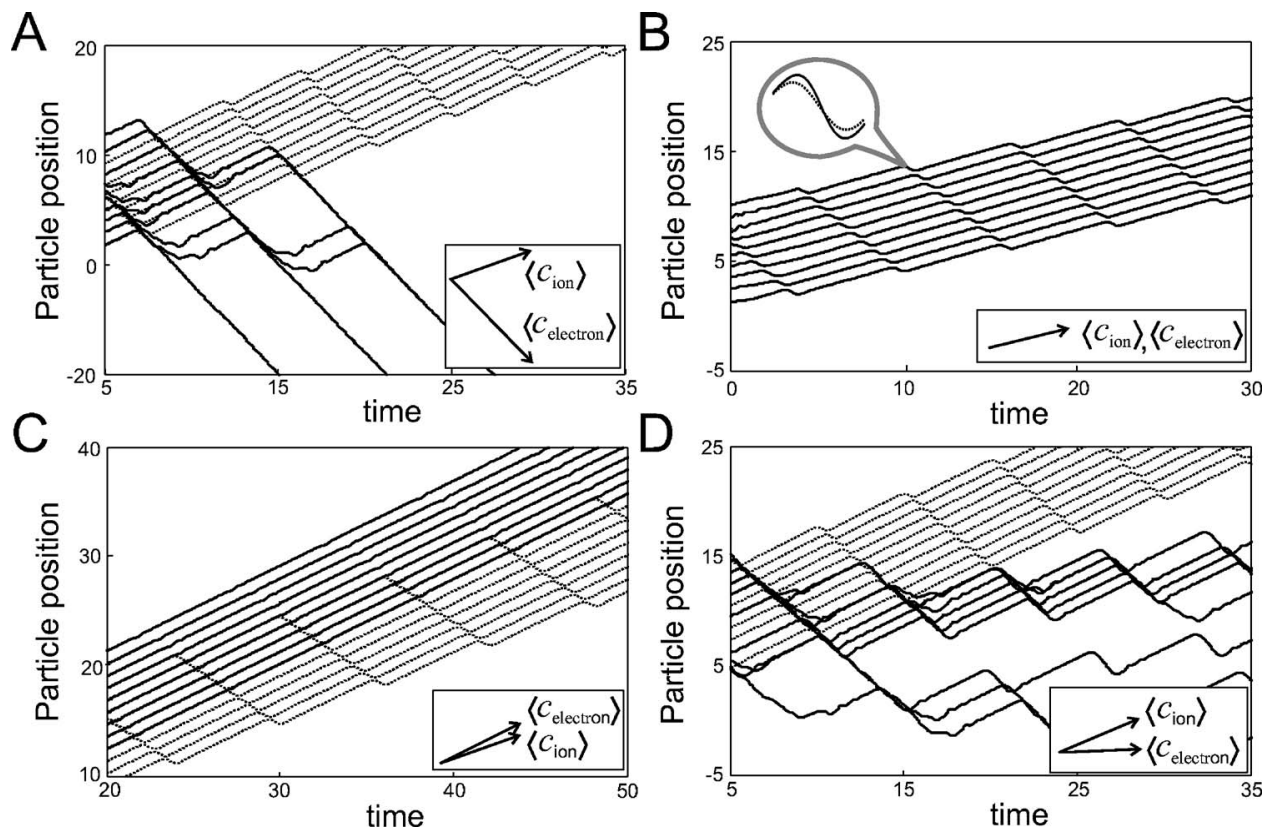

FIG. 6. Solectrons and ion-electron bound states. Lattice ion trajectories (mostly progressing clockwise dotted lines) exhibiting soliton waves (progressing counterclockwise motions along the negative slopes of the ion trajectories) and electron trajectories (thicker lines). Most of the time ions go clockwise (downstream) with positive velocity (positive slope of the trajectories). Then one at a time, and subsequently all one after another in periodic sequence move backwards for a short time interval, defining the portions of trajectories with negative slope. The counterclockwise (upstream) wave motion along the negative slope delineates the path of the soliton wave in the ion lattice. Electrons exhibit different behaviors: (A) soliton-trapping ( $\mu=0.6, B=7)$; (B) ion-trapping $(\mu=0.2, B=6)$; (C) interion jumps ( $\mu=0.6, B=3.8)$; (D) saccadic motions with alternance of soliton- and ion-electron trappings $(\mu=0.6, B=5.5)$. Dimensionless quantities are according to Eq. (11).

compression in the anharmonic lattice once excited rotates in the ring independently on the electric field, provided the field strength is moderate enough. Indeed, as the field strength becomes high enough (of the order $1 / \varepsilon$ ) the wave originated in the ion lattice vibrations would be altered and eventually destroyed. Due to the ion-electron interaction we can say that the lattice vibrations induce an additional "inner" field (in the approximation $\varepsilon \rightarrow 0$ ) with a time-dependent potential for the electrons. Figure 5 shows two snapshots of such potential landscape due to a single-peak soliton $(m=1)$ mode. The deepest minimum, corresponding to a local compression in the lattice, moves with the wave velocity, $c_{\text {wave }}$, say, counterclockwise (to the left). Then the minima formed by the other, nearby "isolated" ions, outside of the compression area, move clockwise with the velocity, $c_{i o n}$.

As earlier shown the velocity of the compression wave increases with the increase of the stiffness of the lattice springs, $B$ (Fig. 5). Upon increasing the stiffness the ions less and less approach each other and the potential well induced by the wave reduces its depth becoming shallower [Fig. 5(B)]. For low enough values of the electric field strength the electrons will interact with this potential and can be trapped by the global, deepest minimum forming a dynamic bound state (solectron). Indeed, after a transient process, the electron moves with the wave (soliton) yielding an electric current independent of the electric field strength and determined solely by the soliton velocity (18), hence by the intrinsic anharmonic lattice dynamics. Note that as the single-peak soliton wave moves the electron changes partners. The solectron is a dynamic entity. It involves a moving soliton, the same from the wave perspective albeit involving different ion clusters along the lattice [18]. Figure 6 shows trajectories of $N=10$ electrons and $N=10$ ions for different values of the parameters of the lattice. All cases refer to a single-peak soliton compression wave in the lattice. There is a regime when all electrons are trapped by the soliton propagating with supersonic velocity [Fig. 6(A)]. The electrons move counterclockwise ("upstream") while ions do it clockwise ("downstream"). The relative velocity of the solectron is obtained by subtracting the velocity of the electrons from that of the ions. We have

$$
\Delta v_{\text {solectron }}=\left\langle v_{\text {ions }}\right\rangle-\left\langle v_{\text {electrons }}\right\rangle=\left(L / T-c_{\text {wave }}\right)-c_{\text {wave }},
$$

where $L$ is the ring length and $T$ is the oscillation period given by Eq. (17). Note that according to Eq. (10), here Eq. (20) gives the current flowing along the ring. Substituting Eqs. (17) and (18) in Eq. (20) we obtain

$$
\Delta v_{\text {solectron }}=3 \frac{L}{\pi} \sin \left(\frac{\pi}{L}\right)\left[1+\sqrt{\mu}\left(\alpha B-\frac{2}{3} \beta\right)\right] .
$$

Thus we have a net counterclockwise, fast upstream electric current, as predicted in Ref. [18]. As the parameters of the system change values in a broad range, a variety of behaviors exists due to the diversity of soliton wave profiles and hence due to the corresponding diversity of potential landscapes offered by the ions with their lattice vibrations to the free electrons. Yet for all these regimes, the qualitative dynamics of the lattice is the same, i.e., a solitonlike local 


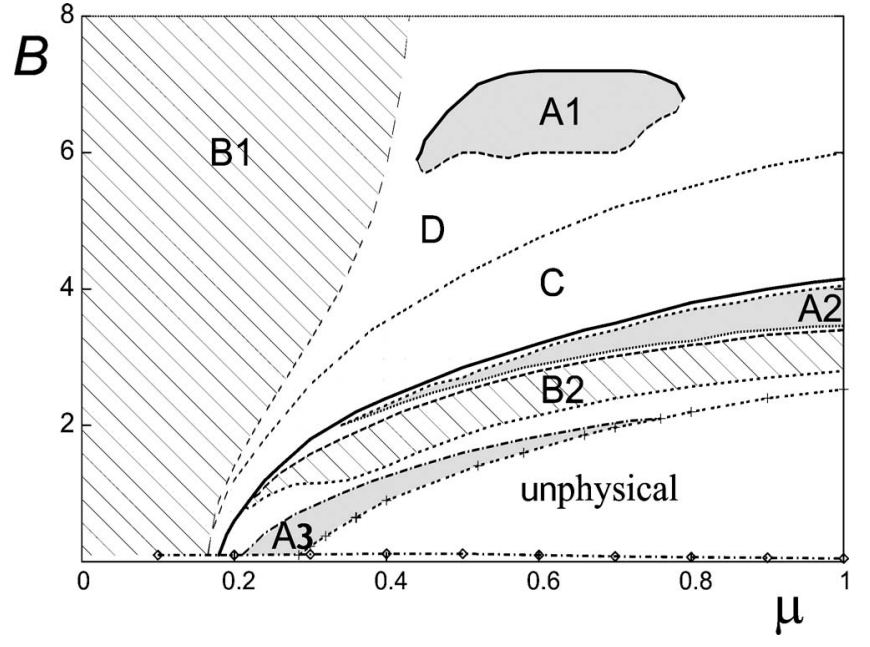

FIG. 7. Nonequilibrium $(B, \mu)$ phase diagram of a drivendissipative anharmonic lattice. It provides the regions with qualitatively different behaviors of the electrons shown in Fig. 6. Areas marked by "A" correspond to the soliton-trapping hence the solectron regime, by " $\mathrm{B}$ " to the ion-trapping, and by " $\mathrm{C}$ " to interion jumps. The unphysical region is an artifact due to the limitation of the exponential interaction as mentioned in the main text. Dimensionless quantities are according to Eqs. (11) and (13).

compression wave propagates in the ring. Figures 6(B)-6(D) show examples of different behaviors taken by the electrons. First we found that electrons can be trapped by ions: an electron stays with an ion and even when the wave passes through this given electron-ion pair remains bound together. We call this the ion-trap case (subscript: iontrp) [Fig. 6(B)]. In this case electrons and ions move in the same direction, with the same mean velocity, and we have no net current:

$$
\Delta v_{\text {iontrp }}=\left\langle v_{\text {ions }}\right\rangle-\left\langle v_{\text {electrons }}\right\rangle=0 .
$$

Figure 6(C) shows another behavior. Between two consecutive passes of the wave each electron is bound to an ion, so we have the ion-trap behavior very much like that shown in Fig. 6(B). However, when the wave arrives the electron is loose and energetic enough to jump to the next ion ahead in the lattice. The jump occurs in the opposite direction of wave propagation. Thus instead of following the wave, like in Fig. 6(A), the electron lags behind it. The wave, although rotating counterclockwise, at each period provokes clockwise jumps of the electrons that move in the same direction (clockwise) to that of the ions but faster. Hence we have a net clockwise (negative), downstream current:

$$
\Delta v_{\text {ionjmp }}=\left\langle v_{\text {ions }}\right\rangle-\left\langle v_{\text {electrons }}\right\rangle=-\frac{L}{N T},
$$

where $N$ is the number of ions in the ring, and $T$ is the oscillation period given by Eq. (17).

Figure 6(D) illustrates a regime where each electron stays for a short time with the wave, then it is trapped by an ion until the next arrival of the wave and then gets trapped by another ion, then by the wave and so on. This sequence repeats albeit not necessarily in a periodic manner. Thus we have a kind of saccadic sequence of soliton-trap and ion-trap regimes shown in Figs. 6(A) and 6(B). The net current (subscript saccadic) in this case depends on the fine tune of the lattice parameters and can be expressed as

$$
\Delta v_{\text {saccadic }}=\left\langle v_{\text {ions }}\right\rangle-\left\langle v_{\text {electrons }}\right\rangle \approx n \frac{L}{N T},
$$

where $n$ is a natural number describing the number of electron-ions jumps over during the wave-trap phase [in Fig. 6(D) $n=2]$.

We have numerically delineated in the parameter space $(B, \mu)$ of the lattice the regions with qualitatively similar behavior (nonequilibrium phase diagram) of the electronwave interactions described above (Fig. 6). Figure 7 summarizes the results. We found three distinct "A" areas (A1, A2, and A3) that correspond to the formation of a solectron [Fig. 6(A)]. The difference between these areas is in the velocity of the solectron and in the resulting current (21). The solectron is supersonic only in the A1 region.

In the approximation of a compression wave of permanent form propagating along the lattice, we can express the motion of the ions by the wave solution $\left\{x_{k}(t)\right\}$. Then Eqs. (12) reduce to

$$
\frac{d y}{d t}=u, \quad \frac{d u}{d t}=-\phi(y, t)-\Gamma u-\mathrm{E},
$$

where $\phi(y, t)=\sum_{k=1}^{N} F\left(y-x_{k}(t)\right)$ is an effective Coulomb force. Equations (25) describe the evolution of free electrons driven by the time-dependent field induced by the ion wave (see the potential landscapes of Fig. 5). For convenience we rewrite Eq. (25) in the form

$$
\begin{gathered}
d y / d t=u, \\
d u / d t=-\frac{\partial \Phi(y, z)}{\partial y}-\Gamma u, \\
d z / d t=c_{\text {ion }}-c_{\text {wave }},
\end{gathered}
$$

where $z$ is an auxiliary variable linearly growing with time. To analyze the dynamics of Eq. (26) we study qualitative changes in a sequence of $2 \mathrm{D}$ sections $(y, u)$ of the phase space taken at constant values of $z$.

Numerical results show that after a short transient process all electrons are trapped by their nearest ions and tend to stay with them, hence moving clockwise with the ions. Then the soliton wave, continuously running counterclockwise, eventually reaches the region where an electron is located. As earlier described, how the electron behaves depends on the parameter values of the system. Three qualitatively different behaviors have been depicted in Figs. 6(A)-6(C). Take the electron bound to the $k$ th ion. Figure 8, column I (case $z$ $=z_{0}$ ), shows the positions of the three nearest ions to the electron, together with the soliton compression wave (upper part of the column), a sketch of the potential landscape $\Phi(y)$, induced by the ion lattice vibrations, and the phase space $(u$, $y$ ) of the electron. The electron is inside the basin of attraction of the $k$ th ion [shaded area in Fig. 8(I)]. Small perturbations decay in time, hence the electron is trapped and dragged by the ion. 


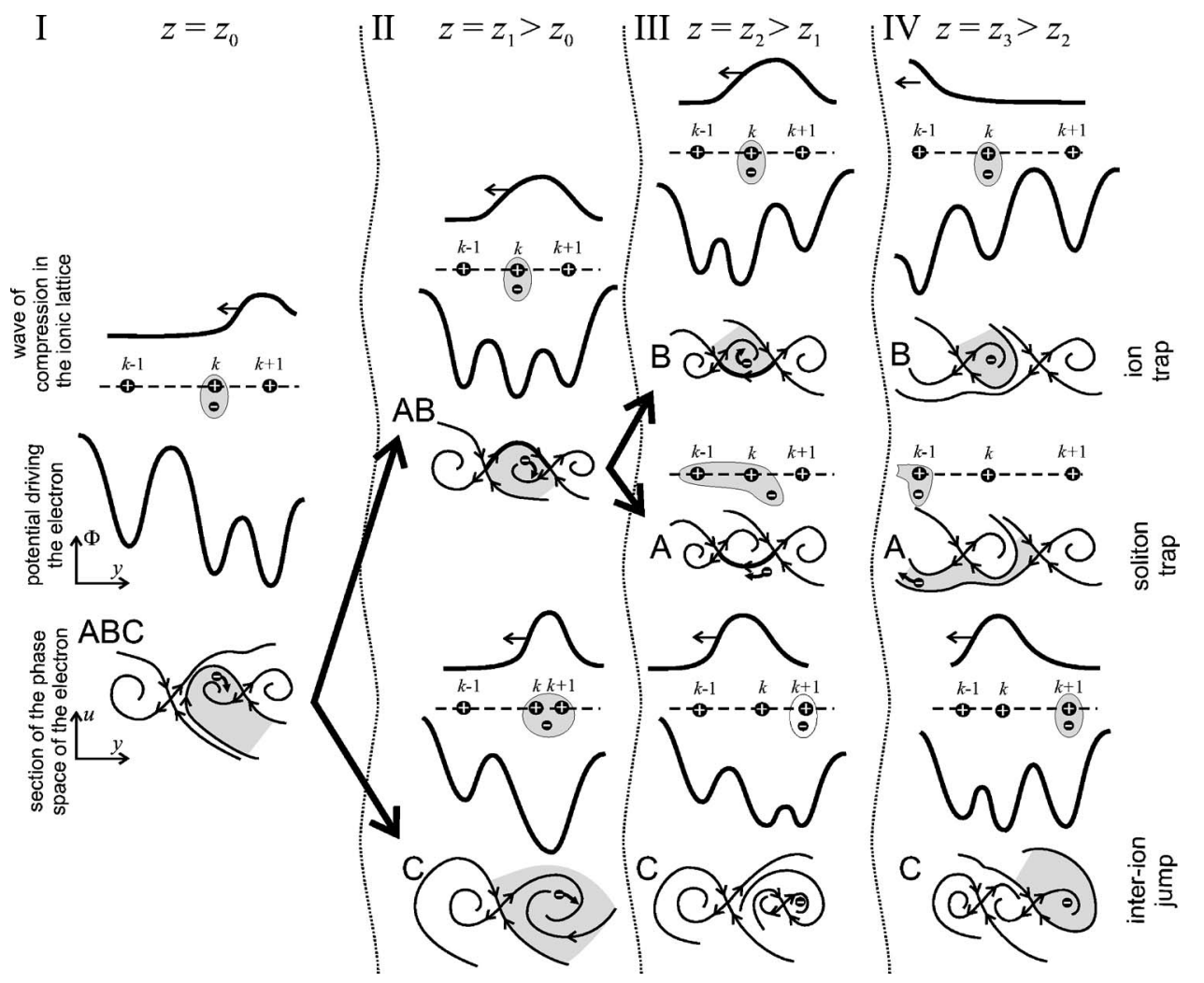

FIG. 8. Lattice dynamical system. Qualitative bifurcation picture and the panoply of behaviors offered by the interaction of an electron (-) with a solitonic compression wave in an anharmonic lattice. Three qualitatively different cases: ion trapping, soliton trapping, and forward interion jump (see also Fig. 6). Depending on the parameter values one or another of these behaviors occurs (see also Fig. 7). (I) Column $z=z_{0}$ shows the time instant just before the wave approaches the electron. The upper part of the column is a snapshot of the ion configuration and the electron position [only the three nearest ions (+) to the electron are shown]. The center part of the column shows a snapshot of the potential landscape corresponding to the ion configuration. The bottom part presents a section of the phase space of the electron taken at $z=z_{0}$. The shaded area delineates the basin of attraction of the steady state corresponding to the $k$ th ion. (II) The column $z=z_{1}$ shows the time instant of the beginning of electron-soliton interaction. Depending on parameter values two qualitatively different scenarios are possible ( $\mathrm{AB}$ and $\mathrm{C}$, respectively). (III) The column $z=z_{2}>z_{1}$ corresponds to the time instant at which the wave passes over the electron. At this time instant depending on the parameter values the scenario AB splits into two, A and B. (IV) The column $z=z_{3}>z_{2}$ depicts the configurations when the wave leaves the electron location. Finally we obtain three qualitatively different scenarios of the electron-wave interaction.

Assume now that the lattice springs are rather stiff, say $B=6.5$. Then even in the compression region of the wave the ions do not get very close together, as they hardly move from their original positions [Fig. 5(B)]. Yet their motion is truly anharmonic. All the time there is a maximum in the potential profile between two neighboring ions [Fig. 8(II), AB]. Consequently, the first bifurcation that occurs is the separatrix loop going from the saddle between the $k-1$ and $k$ ions to the saddle between the $k$ and $k+1$ ions [Fig. 8(II), AB, thicker line]. Further, the distance between the saddles decreases even more and a second bifurcation occurs. Now the separatrix loop goes from the saddle between the $k$ and $k$ +1 ions back to the saddle between the $k-1$ and $k$ ions [Fig. 8(III), A and B]. At this point the motion of the electron is determined by the kinetic energy it had before, or in other words, by the relative velocity of the soliton wave and the ion velocity in the third equation of Eq. (26). For a low energy level, i.e., low values of $\mu$ (domain B1 in Fig. 7), the electron does not have enough energy to leave the potential well and oscillates there staying in the attraction area of the $k$ th ion [Fig. 8(III), B]. When the wave leaves the area of the electron location, the electron remains bound to the ion [Fig. $8(I V), B]$. Thus we have the dynamical regime called ion trapping [Fig. 6(B)].

If $\mu$ is increased enough (like in domain A1 in Fig. 7) the electron goes out of the attraction area and ends below the separatrix loop [Fig. 8(III), A]. Then the electron follows the trajectory towards the $k-1$ ion [Fig. 8(IV), A], hence following the wave. In a certain range of parameter values (A1 in Fig. 7) the dynamic balance between the energy obtained from the wave and the energy dissipated by the electron (controlled by $\gamma_{e}$ ) ensures that the electron experiences a "free" ride while staying always between separatrices like in the phase plane in Fig. 8(IV), A, and is never trapped by an ion. Accordingly, we have the solectron, i.e., the electron is bound to the soliton [Fig. 6(A)].

Let us now consider the case of softer springs, say $B$ $=3.8$. With such stiffness, several ions will closely approach each other and at some section $z=z_{1}$ the maximum in the potential profile between the ions $k$ and $k+1$ disappears [Fig. 

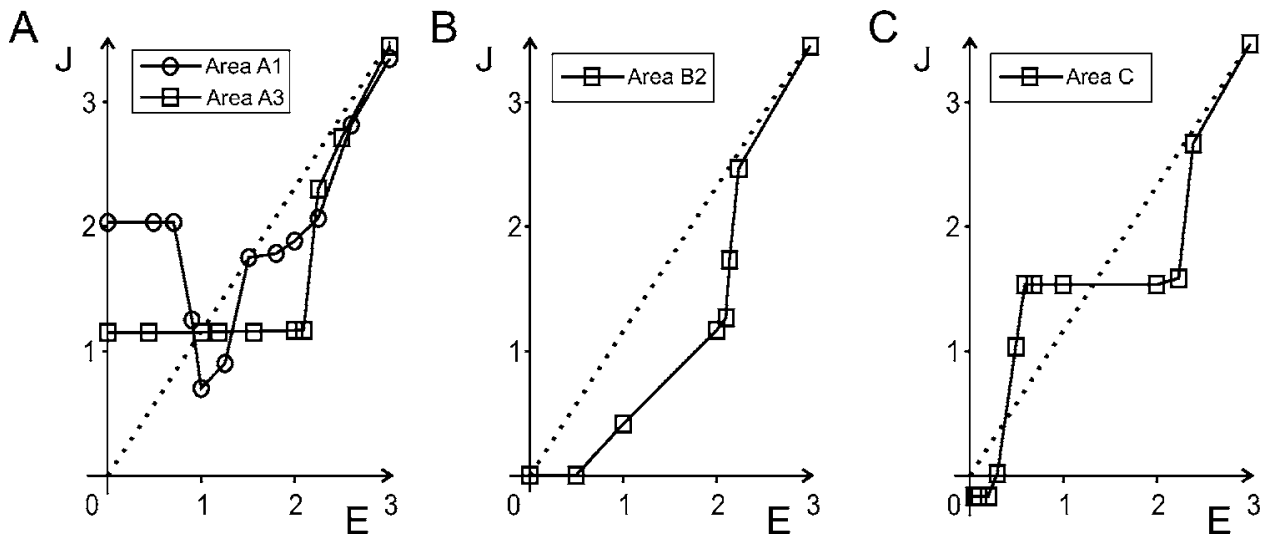

FIG. 9. Current-field (voltage drop) characteristics for an anharmonic lattice with ions and free electrons. Due to anharmonicity the ion vibrations lead to soliton waves propagating along the lattice. These soliton waves mediate the electron-ion interactions and hence determine the electric current. Depending on parameter values (see Fig. 7) the electric current follows Ohm's law (Drude conduction) or becomes non-Ohmic. The latter is clearly a consequence of the anharmonicity. Its actual value depends on the stiffness of the springs. The result also depends on the input-output energy balance set by the active friction maintaining the nonlinear oscillations and corresponding solitonic waves. (A) Solectron areas $(\mu=0.5, B=1.5$ or $B=6.5)$; (B) ion trap $(\mu=0.5, B=2.2)$; (C) interion jumps $(\mu=0.5, B=3.6)$. In all cases the dotted line shows Ohm's law. Dimensionless quantities are according to Eqs. (10) and (13).

8(II), C]. This corresponds to a pitchfork bifurcation, i.e., three steady states merge forming one. The electron follows the trajectory, so when the wave passes further, a new inverse pitchfork bifurcation occurs and three steady states appear again. Now the electron ends in the basin of attraction of the $(k+1)$ th ion [Fig. 8(III), C] and then follows it [Fig. $8(\mathrm{IV}), \mathrm{C}]$. Thus as the wave passes it carries the electron to the next ion ahead, and so on; we get the interion jump regime [Fig. 6(C)].

The different interaction scenarios of an electron with a soliton will lead to qualitatively different current-voltage characteristics. A weak field cannot destroy the bound states described above [Figs. 6 and 8]. Thus the current induced by the anharmonic wave in the ion lattice and consecutive interaction with free electrons will not depend on the value of $E$. The exact value of the electric field independent current is defined by either of Eqs. (21)-(23). Figure 9 shows examples of current-field characteristics for these three cases. Formation of a solectron [Fig. 9(A)] results in a high amplitude constant current even for vanishing electric field strength. Such a constant value is different for different parameter regions, A1, A2, and A3 (Fig. 7).

However, when the electric field is strong enough (applied in either direction), electrons cannot be hold by the lattice wave anymore, and hence they follow the electric field. They start rotating in the ring with the velocity defined by the ratio $E / \Gamma$, which gives rise to the Drude current following Ohm's linear conduction law. For high values of the electric field strength the anharmonicity of lattice vibrations is not strong enough to dominate the dynamics. Clearly, we are outside the domain of validity of the assumption used in Sec. III, following the derivation of Eqs. (12), and the current satisfies Ohm's law. Figures 9(B) and 9(C) illustrate two other regimes with vanishing and negative current, respectively, when the field strength becomes very weak as given by Eqs. (22) and (23) [see Figs. 6(B) and 6(C)].

\section{SUMMARY OF RESULTS}

In view of the possibility of using natural or synthetic molecules (polypeptide chains are one such case) for electronics we have considered here a paradigmatic 1D charged lattice model with anharmonic (exponentially repulsive) dynamics allowing for soliton-mediated Ohmic and non-Ohmic electric transport. We have shown that solitons in such an anharmonic driven-dissipative system can be sub- or supersonic. The supersonic regime occurs for high values of the stiffness in the lattice leading to high compressions.

There are several qualitatively different types of interaction between a soliton wave in the ion lattice and electrons: (i) The solectron regime: the electron is trapped by an upstream running soliton while ions move downstream; (ii) Partial trapping by an upstream running soliton. Although electrons move upstream with the soliton, from time to time they move downstream with ions. (iii) Complete trapping by the downstream moving ions. (iv) Downstream jumps of electrons between ions induced by each pass of the soliton wave (electrons move with ions, but when the soliton arrives at an electron this jumps downstream to the next ion, so the mean velocity of the electrons is higher than that of ions). The solectron regions and hence the regions of anharmonically driven fast electric conduction have been delineated in the nonequilibrium $(B, \mu)$ phase diagram.

\section{ACKNOWLEDGMENTS}

The authors acknowledge fruitful discussions with Dr. Ezequiel del Rio, Dr. Dirk Henning, Dr. Jeff Porter, Dr. Christian Neissner, Professor Jens Feder, Professor Gregoire Nicolis, Professor Gerd Röpke, and Professor Bo Thide. This research has been sponsored in part by the European Union under SPARK (Grant No. FP6-2003-IST-004690) and by the Spanish Ministerio de Educacion y Ciencia under a Ramon y Cajal grant (awarded to V.A.M.). 


\section{APPENDIX: DERIVATION OF THE OSCILLATION PERIOD}

We start with Eq. (14) assuming that $\mu \ll 1$ is a smallness parameter. For $\mu$ positive (just after the Hopf bifurcation) we have low amplitude oscillations whose frequency in first approximation is

$$
\omega=\frac{2 \pi}{T(0)}=2 \sin (\pi / \lambda),
$$

where $T$ is the period of linear oscillations given by

$$
T_{m}(0)=\frac{\pi}{\left|\sin \frac{\pi m}{N}\right|} .
$$

$\lambda=N / m$ is the wavelength $(m=1$ corresponds to a singlepeak soliton).

We seek a solution of Eq. (14) in the form of a running harmonic wave:

$$
x_{k}=\frac{a \sqrt{\mu}}{\omega} \cos \theta, \quad v_{k}=-a \sqrt{\mu} \sin \theta,
$$

with $\theta=\omega t+M k+\phi$, and $M=2 \pi / \lambda ; a$ and $\phi$ are the amplitude and phase of the oscillations, in general slow functions of time.

Changing the variables $\left(x_{k}, v_{k}\right)$ in Eq. (14) by $(a, \phi)$, and expanding the exponentials up to terms of the order of $\mu^{3 / 2}$, we obtain

$$
\begin{aligned}
& \frac{d a}{d t}=-\frac{a \omega}{2} \sin 2 \theta-\frac{\Delta S+G}{\sqrt{\mu}} \sin \theta, \\
& \frac{d \phi}{d t}=-\omega \cos ^{2} \theta-\frac{\Delta S+G}{a \sqrt{\mu}} \cos \theta .
\end{aligned}
$$

with

$$
\begin{aligned}
\Delta S= & -\frac{a \sqrt{\mu}}{3 \omega^{3}} \sin ^{2}(M / 2)\left[3\left(a^{2} B^{2} \mu+4 \omega^{2}\right) \cos \theta\right. \\
& -4 a^{2} B^{2} \mu \cos M \cos ^{3} \theta+a^{2} B^{2} \mu \cos 2 M \cos 3 \theta \\
& +6 a B \sqrt{\mu} \omega \sin M \sin 2 \theta], \\
& \quad G=a \gamma \mu^{3 / 2} \sin \theta\left(a^{2} \sin ^{2} \theta-1\right) .
\end{aligned}
$$

Note that the expansion is valid if $\epsilon=\sqrt{\mu} B \ll 1$, hence this quantity should be considered the new smallness parameter of the problem. Now averaging these equations over the fast variable $\theta$ and substituting Eq. (A1) $(\omega=2 \sin M / 2)$ we obtain

$$
\begin{gathered}
\frac{d a}{d t}=\frac{\gamma \mu a}{8}\left(4-3 a^{2}\right), \\
\frac{d \phi}{d t}=\frac{a^{2} B^{2} \mu}{8} \sin (M / 2) .
\end{gathered}
$$

Equations (A7) and (A8) define a dynamical system in polar

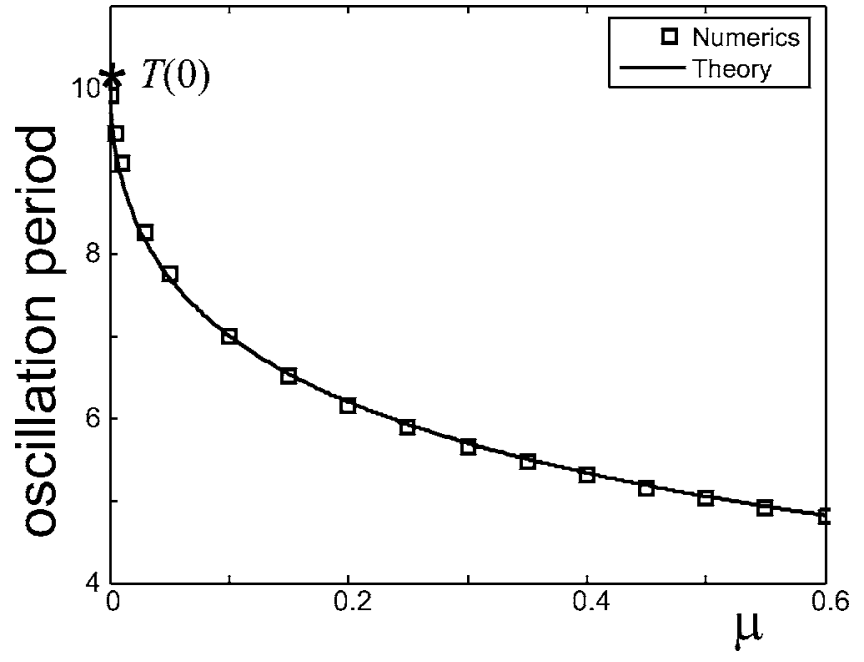

FIG. 10. Oscillation period as a function of $\mu$. Squares show results of numerical integration of Eq. (14). Solid line corresponds to the fitting by Eq. (A14). Star at $\mu=0$ marks the period of linear oscillations $T(\mu=0)=\pi / \sin (\pi / \lambda) \approx 10.16$. Dimensionless quantities are according to Eqs. (13) and (A14).

coordinates that has an unstable focus in the origin leading to a stable limit cycle of amplitude $a^{*}=2 / \sqrt{3}$. The phase velocity on the limit cycle is

$$
\frac{d \phi}{d t}=\frac{\mu B^{2}}{6} \sin \frac{M}{2}
$$

Going back to the wave solution Eq. (A3), Eq. (A9) defines the first-order correction to the oscillation frequency (A1). Thus for small $\sqrt{\mu} B$ the oscillation period scales as

$$
T \approx \frac{\pi}{\sin (\pi / \lambda)\left(1+\mu B^{2} / 12\right)} .
$$

According to Eq. (A10) for $\epsilon=0$ the oscillation period is given by the linear oscillations, and it decays as $\left(1-\mu B^{2}\right)$ for positive $\mu$.

Let us now consider moderate values of $\mu$. According to the numerical results presented in Fig. 3, with appropriate variable rescaling, the wave profile is almost the same in a wide range of values of the lattice parameters $(B, \mu)$. Using Eqs. (14) and (16) we get an equation describing the wave profile:

$$
\frac{d p}{d \xi}=T \mu\left[\frac{e^{-\sqrt{\mu} B T z}-e^{-\sqrt{\mu} B T z\left(\xi+\lambda^{-1}\right)}}{\mu^{3 / 2} B}+\gamma p\left(1-p^{2}\right)\right] .
$$

The bracketed quantity on the right-hand side of Eq. (A11) should scale as $(\mu T)^{-1}$. Then the difference in the exponents scales as $\mu^{3 / 2} B^{1} \gamma \psi(\xi)+T^{-1} \mu^{1 / 2} B \phi(\xi)$, where $\psi$ and $\phi$ are periodic functions depending on the running coordinate $\xi$ only. On the other hand, a modified cnoidal wave solution of Eq. (A11) can be written as 


$$
\begin{aligned}
e^{-\sqrt{\mu} B T z(\xi)}-1= & \frac{4 K^{2}}{T^{2}}\left\{d n^{2}\left[2 K\left(\xi-\frac{1}{2 \lambda}\right)\right]-\frac{E}{K}+a\right\} \\
& +\mu^{3 / 2} B^{1} \gamma \psi\left(\xi-\frac{1}{2 \lambda}\right),
\end{aligned}
$$

where $K$ and $E$ denote the complete elliptic integrals of the first and second kind, respectively, and $d n$ is the Jacobian elliptic function of modulus $n$. Using this ansatz (A12) we obtain that the modulus $n$ is a constant independent of $\mu$ and $B$. Yet it is to be adjusted in such a way that Eq. (A12) fits the waveform of the numerical solution (Fig. 3). Then the wave period scales as

$$
T \propto \mu^{-1 / 2} B^{-1} .
$$

This approximation is valid for moderate $\epsilon$ but it diverges at $\epsilon \rightarrow 0$. Matching these two asymptotic results we obtain an approximate value of the oscillation period:

$$
T \approx \frac{T(0)}{1+\alpha \sqrt{\mu B}},
$$

where $\alpha$ is a constant to be determined by fitting the theoretical curve (A14) to the numerical results. Substituting Eq. (A2) into Eq. (A14) we end up with the estimate (17). Figure 10 shows the oscillation period found by direct integration of the governing equations (open squares) and the theoretical estimate (solid line), Eq. (A14). The best fit is achieved for $\alpha=0.2387$.
[1] D. DeVault, Quantum-Mechanical Tunneling in Biological Systems (Cambridge University Press, Cambridge, UK, 1984).

[2] A. M. Kuznetsov, Charge Transfer in Physics, Chemistry and Biology: Physical Mechanisms of Elementary Processes and an Introduction to the Theory (Taylor and Francis, London, UK, 1995), and references therein.

[3] J. N. Onuchic, D. N. Beratan, J. R. Winkler, and H. B. Gray, Annu. Rev. Biophys. Biomol. Struct. 21, 349 (1992).

[4] H. B. Gray and J. R. Winkler, Annu. Rev. Biochem. 65, 537 (1996).

[5] J. J. Regan and J. N. Onuchic, Adv. Chem. Phys. 107, 497 (1999).

[6] A. A. Stuchebrukhov, Adv. Chem. Phys. 118, 1 (2001).

[7] H. B. Gray and J. R. Winkler, Proc. Natl. Acad. Sci. U.S.A. 102, 3534 (2005), and references therein.

[8] A. S. Davydov, Solitons in Molecular Systems, 2nd Edition (Reidel, Dordrecht, 1991).

[9] Davydov's Soliton Revisited. Self-Trapping of Vibrational Energy in Protein, edited by P. L. Christiansen and A. C. Scott (Plenum, New York, 1990).

[10] A. C. Scott, Phys. Rep. 217, 1 (1992).

[11] Proton Transfer in Hydrogen-Bonded Systems, edited by T. Bountis (Plenum, New York, 1992).

[12] D. Hennig, Phys. Rev. E 62, 2846 (2000).

[13] D. Hennig, E. B. Starikov, J. F. R. Archilla, and F. Palmero, J. Biol. Phys. 30, 227 (2004).

[14] M. G. Velarde, W. Ebeling, D. Hennig, and C. Neissner, Int. J. Bifurcation Chaos Appl. Sci. Eng. 16, 1035 (2006).

[15] D. Hennig, C. Neissner, M. G. Velarde, and W. Ebeling, Phys. Rev. B 73, 024306 (2006).

[16] A. J. Heeger, S. Kivelson, J. R. Schrieffer, and W.-P. Su, Rev. Mod. Phys. 60, 781 (1988).

[17] Solitons and Polarons in Conducting Polymers, edited by L. Yu (World Scientific, Singapore, 1988).

[18] M. G. Velarde, W. Ebeling, and A. P. Chetverikov, Int. J. Bifurcation Chaos Appl. Sci. Eng. 15, 245 (2005).

[19] A. P. Chetverikov, W. Ebeling, and M. G. Velarde, Contrib. Plasma Phys. 45, 275 (2005).

[20] A. P. Chetverikov, W. Ebeling, and M. G. Velarde, Int. J. Bifurcation Chaos Appl. Sci. Eng. (to be published).

[21] V. A. Makarov, W. Ebeling, and M. G. Velarde, Int. J. Bifurcation Chaos Appl. Sci. Eng. 10, 1075 (2000).
[22] V. A. Makarov, E. del Rio, W. Ebeling, and M. G. Velarde, Phys. Rev. E 64, 036601 (2001).

[23] A. P. Chetverikov, W. Ebeling, and M. G. Velarde, Eur. Phys. J. B 44, 509 (2005).

[24] A. P. Chetverikov, W. Ebeling, and M. G. Velarde, Eur. Phys. J. B 51, 87 (2006).

[25] C. Christov and M. G. Velarde, Physica D 86, 323 (1995).

[26] V. I. Nekorkin and M. G. Velarde, Synergetic Phenomena in Active Lattices. Patterns, Waves, Solitons, Chaos (SpringerVerlag, Berlin, 2002).

[27] M. G. Velarde, Int. J. Quantum Chem. 98, 272 (2004).

[28] M. Ross and F. H. Ree, J. Chem. Phys. 73, 6146 (1980).

[29] M. Ross, F. H. Ree, and D. A. Young, J. Chem. Phys. 79, 1487 (1983).

[30] W. J. Nellis, N. C. Holmes, A. C. Mitchell, R. J. Trainor, G. K. Governo, M. Ross, and D. A. Young, Phys. Rev. Lett. 53, 1248 (1984).

[31] M. Ross and D. A. Young, Phys. Lett. A 118, 463 (1986).

[32] E. M. Apfelbaum, V. S. Vorobev, and G. A. Martynov, J. Phys. Chem. A 108, 10381 (2004).

[33] M. Toda, Nonlinear Waves and Solitons (Kluwer, Dordrecht, 1983).

[34] T. Pohl, U. Feudel, and W. Ebeling, Phys. Rev. E 65, 046228 (2002).

[35] N. Ashcroft and N. D. Mermin, Solid State Physics (Holt, Rinehardt \& Winston, Philadelphia, 1976).

[36] V. Heine, M. L. Cohen, and D. Weaire, The Pseudopotential Concept (Academic Press, New York, 1970).

[37] J. W. Strutt [Lord Rayleigh], Philos. Mag. 15, 229 (1883).

[38] J. W. Strutt [Lord Rayleigh], The Theory of the Sound (Dover reprint, New York, 1941) Vol. I, Sec. 68a.

[39] B. Van der Pol, Philos. Mag. 2, 978 (1926); 3, 65 (1927).

[40] F. Schweitzer, Brownian Agents and Active Particles. Collective Dynamics in the Natural and Social Sciences (SpringerVerlag, Berlin, 2003).

[41] W. G. Hoover, Time Reversibility, Computer Simulation, and Chaos (World Scientific, Singapore, 1999).

[42] R. Klages, K. Rateitschak, and G. Nicolis, Phys. Rev. Lett. 84, 4268 (2000).

[43] K. Rateitschak, R. Klages, and W. G. Hoover, J. Stat. Phys. 101, 61 (2000).

[44] Yu. A. Kuznetsov, Elements of Applied Bifurcation Theory (Springer-Verlag, Berlin, 1995). 